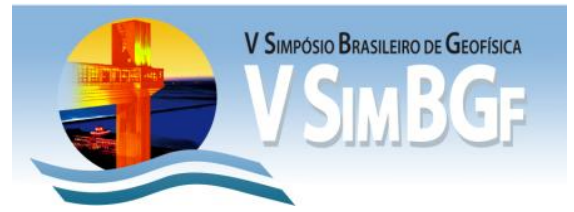

\title{
Atenuação de Reflexões múltiplas do fundo do Mar
}

Marcos Augusto Lima da Luz ${ }^{* 1}$ - marquinholuz@yahoo.com.br

Prof ${ }^{\text {. }}$. Dr ${ }^{\mathrm{a}}$. Rosangela Corrêa Maciel ${ }^{1,2}$ e Prof. Dr. Walter Eugênio de Medeiros ${ }^{1,2}$

${ }^{1}$ Universidade Federal do Rio Grande do Norte - UFRN - ${ }^{2}$ INCT-GP, Instituto Nacional de Geofísica do Petróleo (CNPQ), Brasil

Copyright 2012, SBGf - Sociedade Brasileira de Geofísica

Este texto foi preparado para a apresentação no V Simpósio Brasileiro de Geofísica, Salvador, 27 a 29 de novembro de 2012. Seu conteúdo foi revisado pelo Comitê Técnico do V SimBGf, mas não necessariamente representa a opinião da SBGf ou de seus associados. É proibida a reprodução total ou parcial deste material para propósitos comerciais sem prévia autorização da SBGf.

\section{Resumo}

$\mathrm{Na}$ aquisição de dados sísmicos marítimos é rotineiro o aparecimento de ruídos causados por múltiplas reflexões ocorridas na lâmina d'água. Devido a necessidade de se obter um melhor imageamento da seção sísmica gerada, estudos vêm sendo desenvolvidos com o objetivo de aumentar a relação sinal/ruído (SNR) e melhorar a resolução vertical dos traços sísmicos aplicando, filtragem de frequência, deconvolução e outros. Sendo assim, este trabalho foi desenvolvido com o objetivo de comparar o desempenho na atenuação de múltiplas obtido com diferentes algoritmos já desenvolvidos para esse fim. Em particular, foram comparados os métodos da deconvolução preditiva de tipo Wiener-Levinson (TWL) e a filtragem $\mathrm{f}-\mathrm{k}$, a qual é utilizada para atenuar ruídos coerentes e preservar os eventos de uma determinada faixa de velocidade horizontal. A princípio, os testes foram aplicados em dados sintéticos. Espera-se com esse trabalho obter resultados que reflitam as limitações e funcionalidades de cada filtro.

\section{Introdução}

No caso de aquisição sísmica marítima, devido ao alto coeficiente de reflexão nas interfaces água/ar e água/fundo do mar, ocorrem eventos como múltiplas reflexões, que acabam mascarando informações importantes. Devido a esse fato muitos algoritmos vêem sendo desenvolvidos com o objetivo de suprimir múltiplas reflexões presentes nos dados sísmicos. A exemplo disso temos a deconvolução preditiva multicanal (DPM) e a filtragem F-K que faz uso das transformadas direta e inversa de Fourier. Onde segundo Yilmaz (2008) ruídos lineares coerentes associados a dispersores secundários podem ser atenuados com o uso desse método.

A deconvolução preditiva para supressão de múltiplas, a qual utiliza o processo de filtragem Wiener-Levinson (WL) (Robinson,1980) é um método estatístico que se baseia no caráter periódico das múltiplas, no entanto essa periodicidade só é preservada no caso de incidência normal, o que torna a deconvolução empregada menos efetiva. Na forma multicanal, a deconvolução WL faz uso da coerência lateral dos eventos refletidos presentes em canais adjacentes e por isto tendem a ser mais eficientes que a forma monocanal (Porsani, 1997).

Algoritmos WL multicanal foram aplicados com sucesso na atenuação de múltiplas em dados sintéticos e reais por Lima, 1999, Bezerra, 2001, Santos Jr., 2002 e Maciel, 2007. Neste trabalho aplicamos a deconvolução preditiva tipo Wiener-Levinson (TWL) multicanal no dado organizado em famílias de afastamento comum (AC). 0 algoritmo tipo Wiener-Levinson aplica a recursão de Levinson diretamente ao traço sísmico gerando e atualizando vetores de erro direto e reverso, onde o vetor de erro direto obtido ao final da recursão constitui o traço sísmico deconvolvido (Santos Jr, 2002; Porsani e Ursin, 2007). Na prática, isto significa efetuar a deconvolução preditiva sem a necessidade de calcular os elementos do filtro ou da autocorrelação ou correlação cruzada dos traços sísmicos. Diferentemente do algoritmo WienerLevinson convencional. Utilizamos algoritmos desenvolvidos e disponibilizados por pesquisadores do CPGG-UFBA.

A filtragem $F-K$ é utilizada para retirar freqüências indesejadas, só que de eventos coerentes que possuem uma determinada faixa de velocidade. Onde a transformada de Fourier decompõe o campo de onda original no domínio $t-x$ (tempo-distância) em suas componentes no domínio F-K (freqüência-número de ondas) constante, de forma em que os dados sísmicos de entrada são transformados em uma imagem de densidade de energia como uma função desses parâmetros. Ambos os métodos foram aplicados em quatro conjuntos de dados sísmicos marinhos sintéticos.

\section{Dados sintéticos}

Os modelos sintéticos desenvolvidos simulam a variação de complexidade geológica do fundo do mar e camada subjacente caracterizadas por:

- MODELO SS - fundo do mar simples com geologia simples; MODELO SC - fundo do mar simples com geologia complexa; MODELO CS-fundo do mar complexo com geologia simples e MODELO CC-fundo do mar complexo com geologia complexa. (fig.1)

Os dados foram simulados utilizando o pacote Cshot do Seismic Unix (SU) mantido pelo consórcio CWP (Center of Wave Phenomena) da Colorado School of Mines. Os parâmetros de aquisição dos dados são apresentados na Tabela1.

Tabela 1 - parâmetros de aquisição

\begin{tabular}{|c|c|}
\hline PARÂMETROS & VALORES UTILIZADOS \\
\hline Intervalo entre os receptores $(\mathrm{m})$ & 26 \\
\hline Intervalo entre tiros $(\mathrm{m})$ & 26 \\
\hline Intervalo de CMP $(\mathrm{m})$ & 13 \\
\hline Número de tiros & 500 \\
\hline Número de canais & 180 \\
\hline Intervalo de amostragem (ms) & 4 \\
\hline Número de amostras & 2001 \\
\hline Afastamento mínimo (m) & 52 \\
\hline Afastamento máximo (m) & 4706 \\
\hline
\end{tabular}




\section{Filtragem DPM}

Baseando-se no modelo convolucional o traço sísmico marítimo sem ruído aditivo pode ser descrito por:

$$
z(t)=p(t) * e(t) * m(t)
$$

onde $z(t)$ é o traço sísmico, $p(t)$ o pulso sísmico considerado invariante no tempo e e $(t)$ representa a resposta impulsional da Terra, que inclui as primárias e as múltiplas internas (Yilmaz, 1989) e $m(t)$ é a sequência geradora de múltiplas do fundo do mar. Dada uma sequência $z(t)$ de comprimento $M$, o filtro preditivo irá comprimi-lo para um comprimento $L$, onde $L$ é a distância de predição. Assim para um caso particular onde $L=1$, a operação é dita deconvolução do pulso fornecendo como traço resultante $\tilde{z}(t)$ idealmente formado pela resposta impulsiva da Terra convolvida com o trem de múltiplas associadas a superfície livre:

$$
\tilde{z}(t)=e(t) * m(t)
$$

Assim $\tilde{z}(t)$ resulta num traço com maior resolução temporal já que o pulso $p(t)$ foi comprimido a uma função delta de Dirac (Robinson e Treitel, 1980). Por outro lado quando $L$ é um valor maior que a unidade, o operador preditivo de erro terá sua ação deslocada no tempo, ou seja, atuará sobre amostras situadas há tempos maiores, esse fato nos permite escolher convenientemente o valor de $L$ de forma que o operador atue na zona de múltiplas, promovendo a atenuação das mesmas (Lima, 1999). Ao aplicarmos o operador preditivo obtemos um traço deconvolvido $\tilde{y}(t)$ idealmente livre das múltiplas, ou seja, o traço resultante representaria a série refletividade convolvida com o pulso sísmico:

$$
\tilde{y}(t)=p(t) * e(t)
$$

Convencionalmente, o algoritmo WL mono ou multicanal resolve um sistema de equações lineares que utiliza a matriz dos coeficientes de autocorrelação do sinal de entrada. Se usada na deconvolução de múltiplas, a matriz terá ainda elementos correspondentes a correlação cruzada entre a saída desejada e o sinal de entrada. A recursão de Levinson é então aplicada para resolver o sistema, cuja solução dos mínimos quadrados são os coeficientes do filtro preditivo WL a ser usado na deconvolução das múltiplas (Lima, 1999). Em algoritmos mais atuais (Porsani e Ursin, 2007) a recursão de Levinson é aplicada diretamente sobre o(s) traço(s) sísmico(s) gerando e atualizando o traço deconvolvido sem calcular os elementos do filtro.

\section{FILTRAGEM F-K}

A filtragem $\mathrm{F}-\mathrm{K}$ foi introduzida com o objetivo de reduzir ruídos indesejáveis dos dados (Embree et al., 1963). Tal técnica possibilitou processar um registro sísmico de modo a preservar sem alteração alguma na banda de frequência, todos os eventos com mergulhos em um dado intervalo, e atenuar uniformemente todos os outros que estão fora desse intervalo específico. A Transformada F-K (também conhecida como a Transformada Dupla de Fourier, equação 1.1) separa as bandas de frequência, auxiliando na distinção entre primárias e múltiplas. O dado no domínio t-x (tempodistância) é corrigido (correção NMO) para ser então transformado para o domínio $\mathrm{f}$ - $\mathrm{k}$ (frequência-número de onda). Neste domínio, os eventos primários e múltiplos são bem separados baseando-se em seus mergulhos, para então anular/atenuar a energia das múltiplas. Os próximos passos consistem em transformar o dado para o domínio original (Transformada de Fourier Inversa, equação 1.2) e remover a correção $\mathrm{NMO}$, lembrando que essa é apenas uma das formas de se aplicar o filtro.

$$
\begin{aligned}
& F(k, \omega)=\int_{-\infty}^{\infty} \int_{-\infty}^{\infty} f(x, t) e^{i k x-i \omega t} d t d x \\
& f(x, t)=\frac{1}{4 \pi^{2}} \int_{-\infty}^{\infty} \int_{-\infty}^{\infty} F(k, \omega) e^{-i k x+i \omega t} d \omega d k
\end{aligned}
$$

\section{Aplicação e Resultados}

\section{Filtragem DPM}

Os passos do processamento aplicado aos dados foram:

- Organização em famílias CMP

- Correção de MMO

- Organização em famílias de AC

- Filtragem DPM

- Correção de MMO Inverso

- $\quad$ Organização em CMP

- Análise de velocidades

- Empilhamento

$O$ dado foi dividido em quatro partes de forma a estimar de maneira mais precisa o período $(P)$ a ser utilizado no cálculo da distância de predição $(L)$. Sabendo que $L$ é um percentual de $P$, assim como o número de coeficientes do filtro $(M)$. O período foi coletado sobre cada painel de AC mínimo de cada conjunto de dados através do "piking" usando como referência a primária do fundo do mar.

A DPM foi aplicada em cada parte separadamente, utilizando cinco canais, $\mathrm{L}=0.90 \mathrm{P}$ e $\mathrm{N}=0.20 \mathrm{P}$ e após a filtragem essas partes foram reagrupadas e prosseguindo o processamento conforme os passos descritos acima.

Analisando as fig. $2 a$ e $2 b$, referente ao painel $A C$ mínimo, antes e após a aplicação da DPM, é possível identificar uma menor efetividade na atenuação de eventos múltiplos, conforme se aumenta a complexidade em cada modelo. Além da atenuação das primárias juntamente com as múltiplas, pois em situações onde a múltipla se sobrepõe a primária o filtro não consegue distinguir entre múltiplas de primárias e acaba atenuando tudo que está dentro área de atuação dos coeficientes do filtro. Sendo assim, foi detectado que o filtro é limitado em várias regiões, como as que possuem sinclinais. Isso ocorre devido a dificuldade de se estimar de maneira mais precisa o período das múltiplas em regiões de sinclinais e anticlinais. Uma forma de contornar esse problema é fazer uma boa estimativa do período das múltiplas e um bom ajuste do número de coeficientes do filtro, de forma a aumentar sua efetividade, pois devido às múltiplas reverberarem e se cruzarem no sinclinal, acabam ficando localizadas muito próximas umas das outras no sismograma e mesmo sem a identificação de todos os períodos das primárias, esses eventos são atenuados de forma satisfatória, pois estão dentro da área de atuação do filtro.

Comparando as fig. 3b, 4b, $5 b$ e $6 b$ referente aos dados empilhados após a DPM, com as fig. 3a, 4a, 5a e 6a originais, detectou-se que conforme aumenta a ordem do 
evento múltiplo se tem uma diminuição de sua amplitude, esse fato ocorre devido esses eventos realizarem grandes percursos antes de seu registro ocorrendo então o fenômeno de divergência esférica. Esse fator proporciona uma impressão errônea da verdadeira atuação do filtro, mas devido às baixas amplitudes geradas com esses longos percursos, tais eventos acabam se misturando com ruídos existentes no dado. Devido a esse problema foi aplicado aos dados originais um ganho exponencial para possibilitar a visualização de todos os eventos múltiplos existentes e demonstrar seus comportamentos com a variação da complexidade.

Nota-se que mesmo com certa limitação, a DPM fornece resultados significativos para atenuação de reflexões múltiplas do fundo mar.

\section{Filtragem F-K}

Os passos do processamento aplicados na filtragem F-K aos dados foram:

- Organização em CMP

- Analise de velocidades - intermediárias

- Correção de NMO

- Filtragem F-K

- Correção de NMO Inverso

- Organização em CMP

- Análise de velocidades

- Empilhamento

Mesmo sabendo da dificuldade encontrada em separar completamente primárias e múltiplas com base no mergulho, pois para que isso aconteça deve-se considerar que a terra não possui variação lateral em profundidade, a filtragem F-K é bem eficiente quando se faz uma nova análise de velocidade para corrigir de NMO os eventos antes de sua aplicação, onde se encontram velocidades intermediárias entre primárias e múltiplas, deixando primárias sobrecorrigidas e múltiplas subcorrigidas, diferenciando bem ambas as reflexões e fazendo com que fiquem alocadas em posições contrárias no painel de espectro de frequência, possibilitando assim a remoção/atenuação dos eventos desejados.

Nos resultados obtidos é possível visualizar nitidamente que a filtragem $\mathrm{F}-\mathrm{K}$ foi efetiva em uma grande região do dado, isso pode ser constatado nas fig. $2 \mathrm{a}$ e $2 \mathrm{c}$ onde temos os painéis em offset referentes ao modelo CC, antes e depois da filtragem. Já na seção empilhada após a filtragem é possível agora verificar a real atuação do filtro F-K, pois mesmo com algumas limitações gerou resultados satisfatórios, vistos na comparação feita entre as fig. $3 c, 4 c, 5 c$ e $6 c$ referentes aos dados empilhados após a filtragem, e as fig. 3a, 4a, 5a e 6a do dado original. Devido existir eventos múltiplos e primários com mergulhos e freqüências similares, muitas reflexões múltiplas não podem ser atenuadas por meio desse filtro.

\section{Conclusões}

Com a aplicação da DPM e a filtragem F-K nos quatro modelos sintéticos foi possível identificar que a DPM possui limitações, devido a sua dependência da capacidade de intervenção do interprete para fazer a discriminação correta entre primárias e múltiplas além de ter como base a afirmativa da periodicidade das múltiplas. A DPM se mostrou mais efetiva nos painéis de afastamentos mais curtos, tendo menor efetividade em painéis de afastamentos longos. De modo que ao se aumentar a complexidade do modelo mais difícil se tornava encontrar 0 período das primárias e consequentemente diminuía a área de atuação do filtro.

A filtragem F-K se mostrou mais eficiente, mas também têm suas limitações, tal como a dificuldade em discriminar entre as velocidades primárias e múltiplas com base no mergulho, como é o caso de reflexões múltiplas de curto período, e pequenos offsets. No caso de múltiplas de curto período, as quais causam um enlarguecimento do pulso da primária, e acabam tendo características bem semelhantes de modo a dificultar sua identificação e separação.

\section{Agradecimentos}

Os autores agradecem à PETROBRAS, FINEP, CNPq e INCT-GP pelo financiamento. Aos pesquisadores do LAGEP-CPGG-UFBA por disponibilizarem os algoritmos usados na DPM.

\section{Referências}

Bezerra, A. C. (2001) Deconvolução preditiva multicanal usando interpolação de traços sísmicos e transformada $\tau$ p, Dissertação de mestrado, Universidade Federal da Bahia, Salvador, Brasil.

Embree, P.; Burg, J. P.; Backus, M. M. Wide-band velocity filtering - the pie slice process. [s.I.]: Geophysics, v. 28, p.948-974, 1963.

Lima, A. P. (1999) Deconvoluções de reflexões múltiplas nos domínios t-x e $\tau$-p, Dissertação de Mestrado, Universidade Federal da Bahia, Salvador, Brasil.

Maciel, R. C., 2007. Deconvolução Preditiva Multicanal de reflexões múltiplas no domínio CRS. Tese de Doutorado, Programa de Pós-Graduação em Geofísica, Universidade Federal da Bahia.

Porsani, M. J., 1997. Deconvolução de múltiplas com filtros Wiener-Levinson multicanais, In: Expanded Abstract, 5th International Congress of The Brazilian Geophysical Society., vol. 1, pp. 196-199, SBGF.

Porsani, M. J. e Ursin, B. (2007) Direct multichannel predictive deconvolution, Geohysics, 72:H11.

Santos Júnior, A. (2002) Deconvolução multicanal utilizando um algoritmo tipo Wiener-Levinson Dissertação de mestrado, Universidade Federal da Bahia, Salvador, Brasil.

Yilmaz, Ö. 1987 Seismic Data Processing, SEG, Tulsa, Oklahoma. 


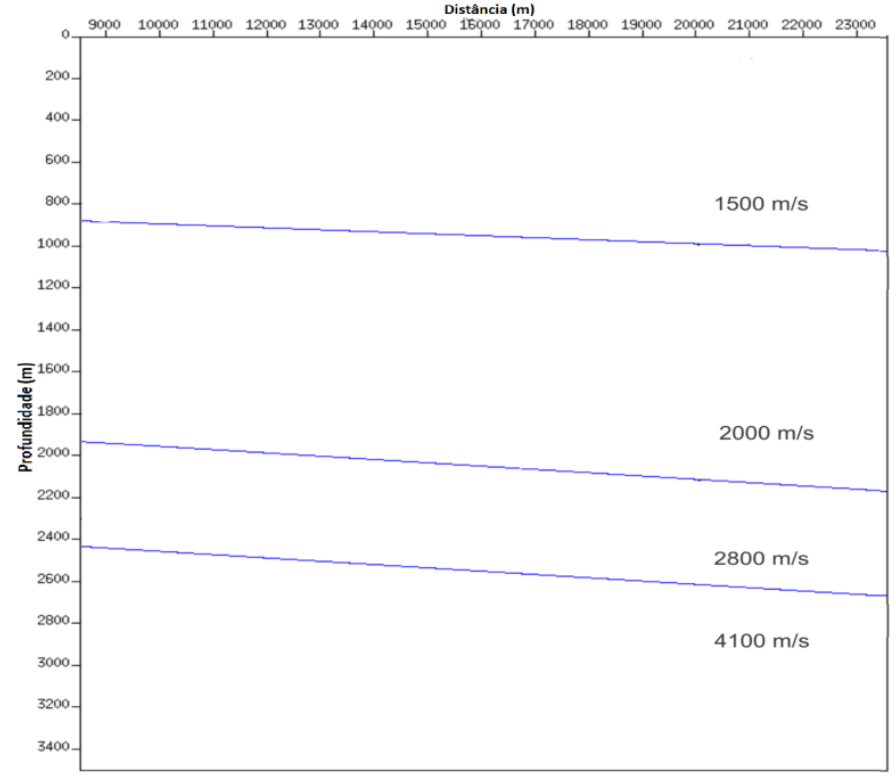

a

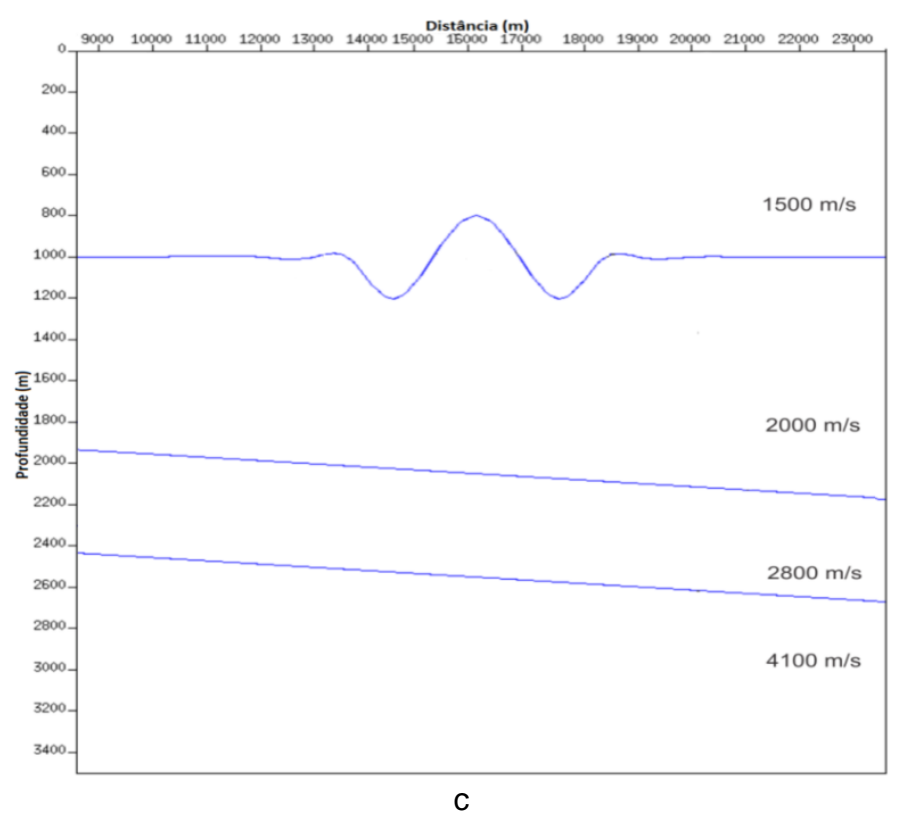

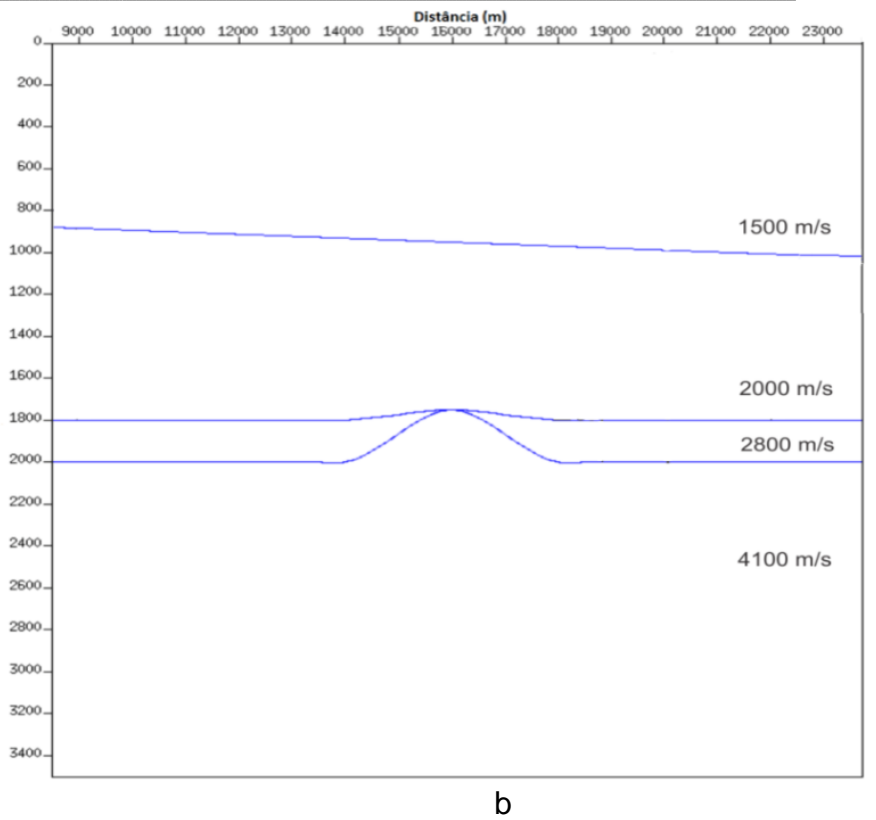

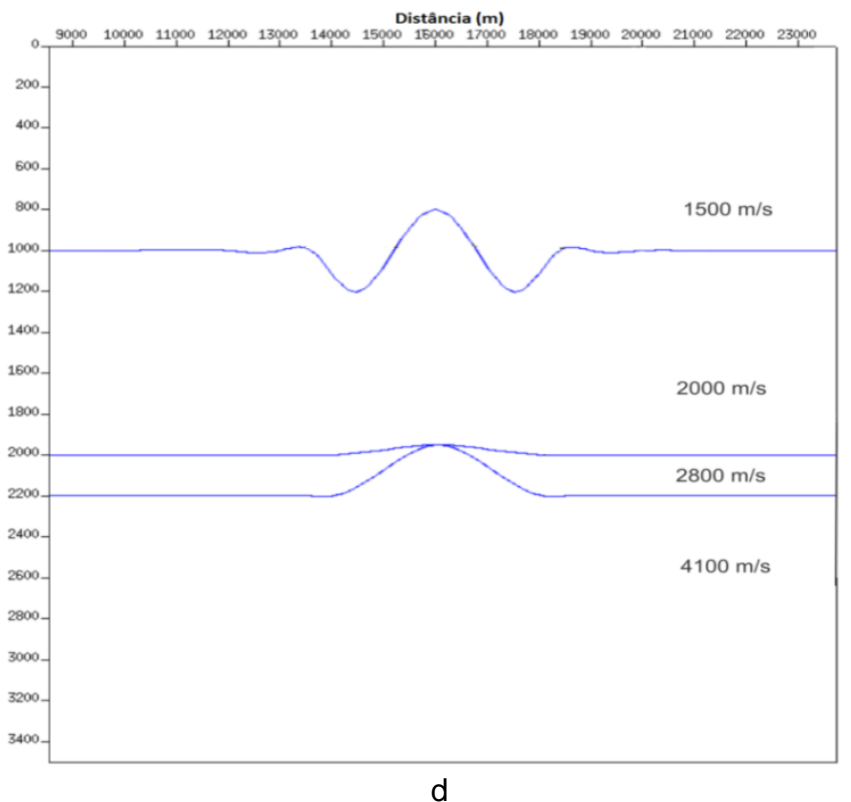

d

Fig. 1: a - MODELO SS; b - MODELO SC; c - MODELO CS e d - MODELO CC.

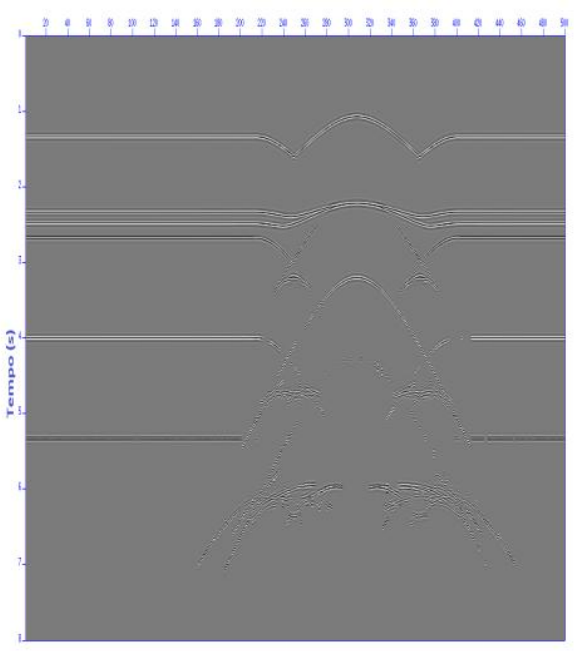

a

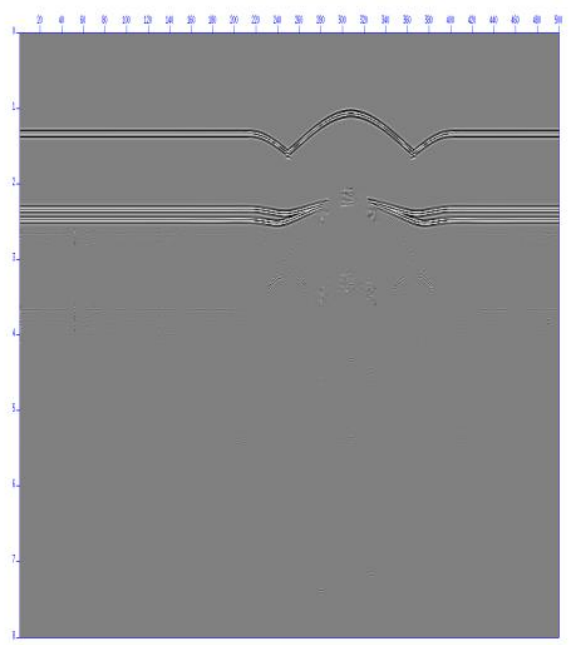

$b$

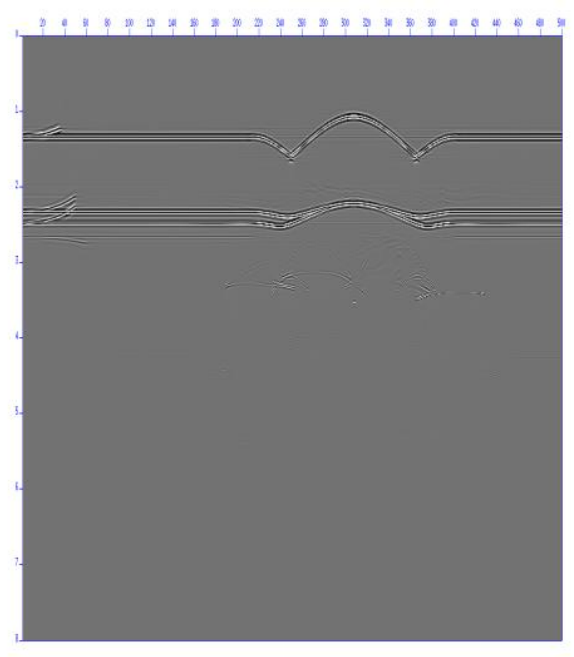

$c$

Fig. 2: Painéis de AC mínimo MODELO CC, a - original; b - após a aplicação da DPM; e c - após a filtragem f-k. 


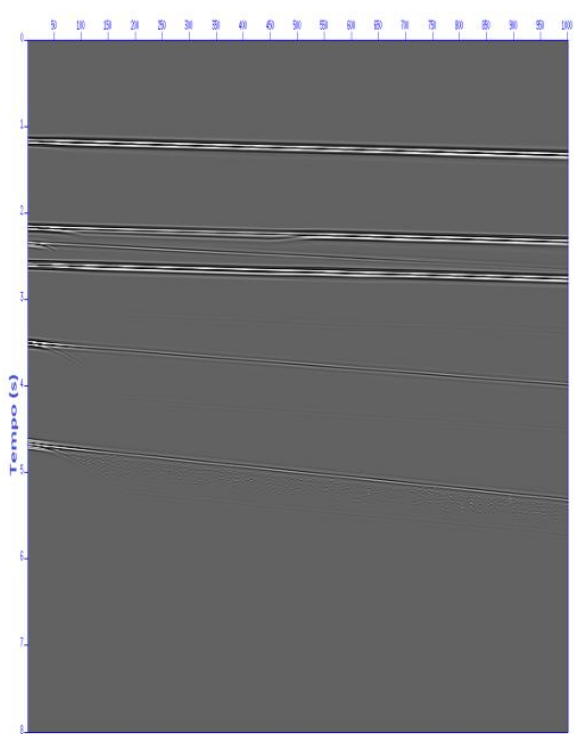

a

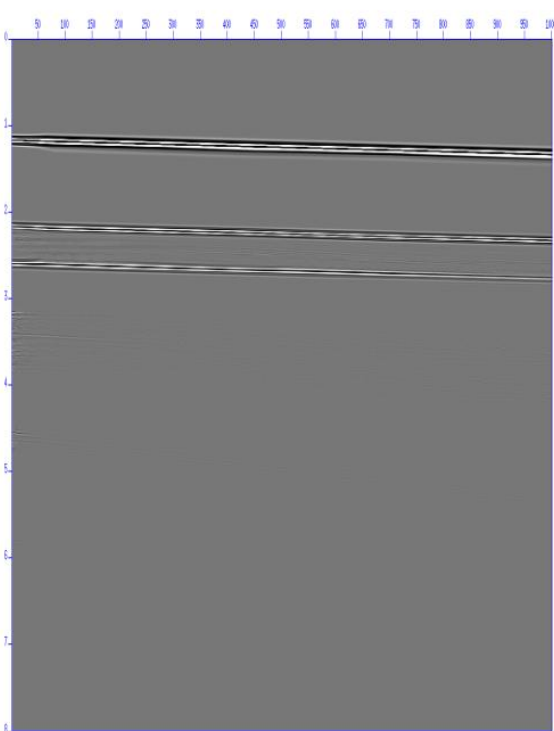

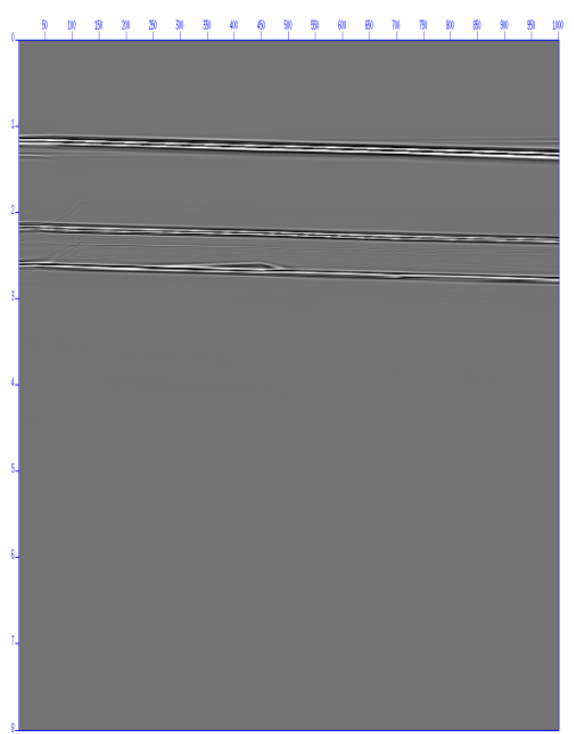

Fig. 3: Seção empilhada MODELO SS, a - original; b - após a aplicação da DPM; e c - após a filtragem f-k.

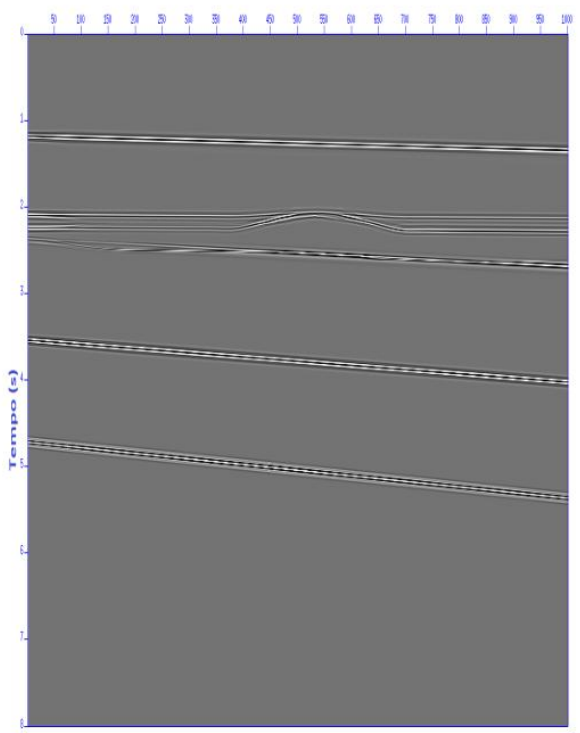

a

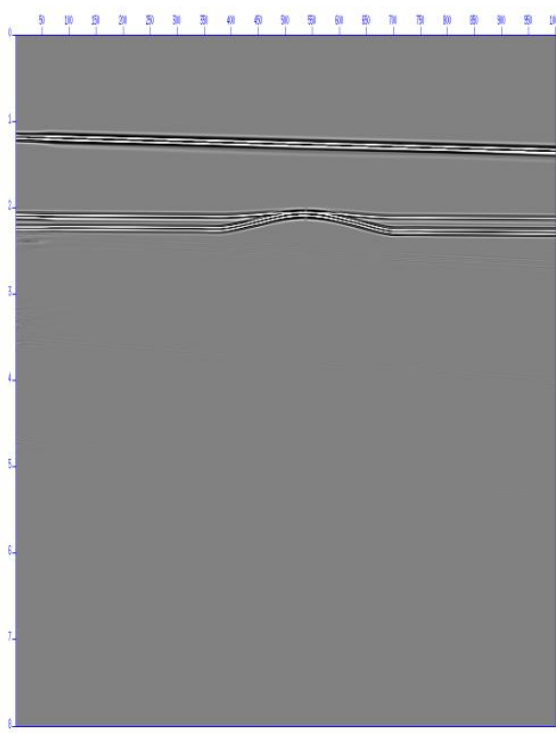

$b$

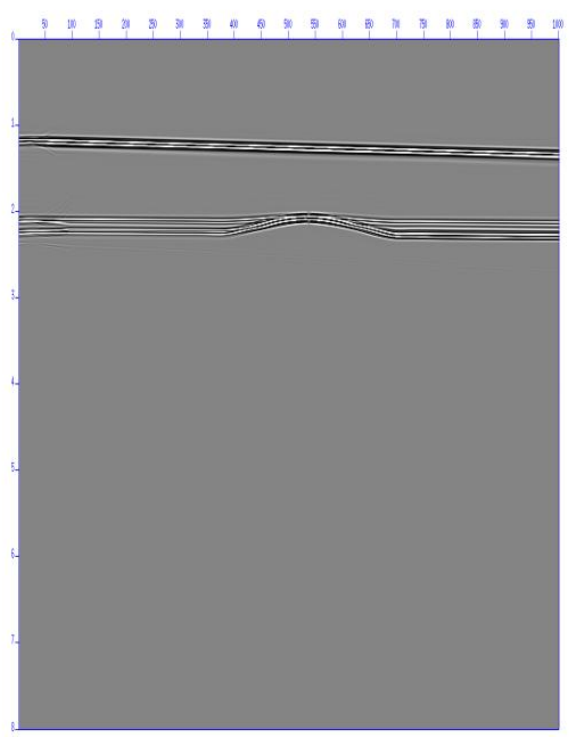

Fig. 4: Seção empilhada MODELO SC, a - original; b - após a aplicação da DPM; e c - após a filtragem f-k. 


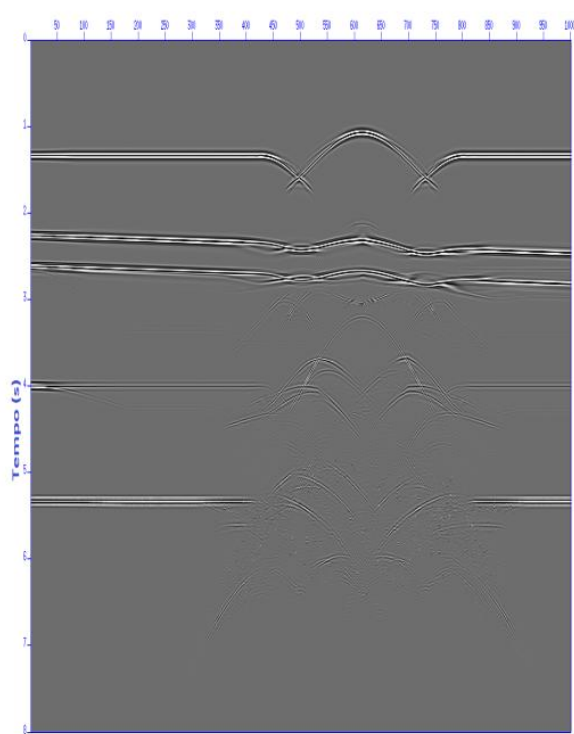

a

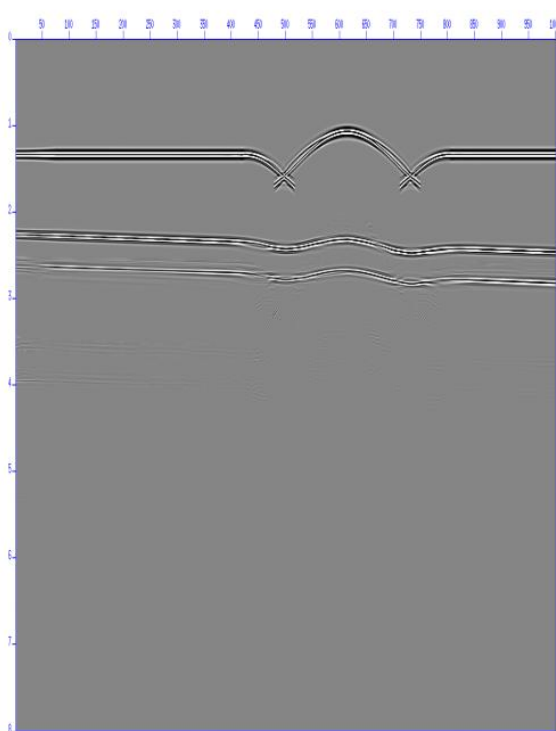

b

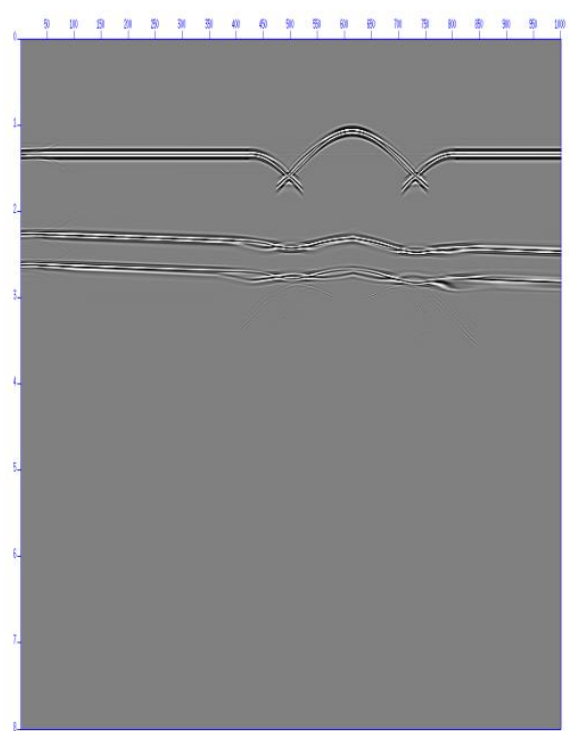

C

Fig. 5: Seção empilhada MODELO CS, a - original; b - após a aplicação da DPM; e c - após a filtragem f-k.

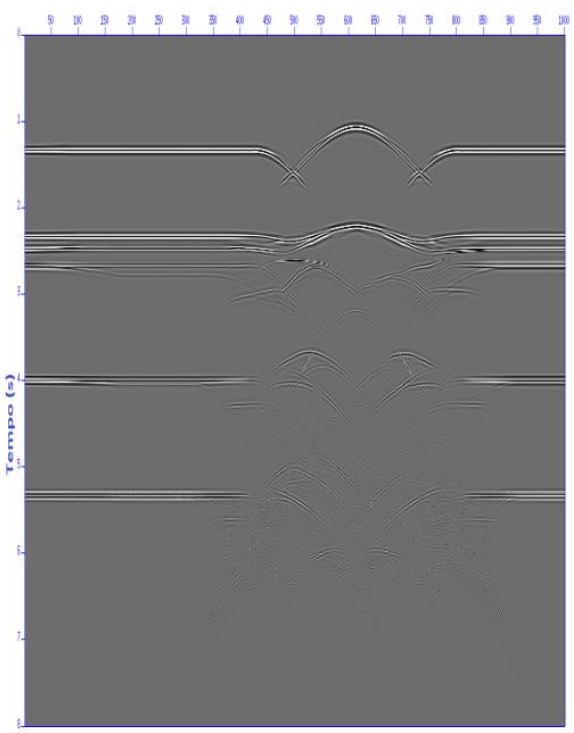

a

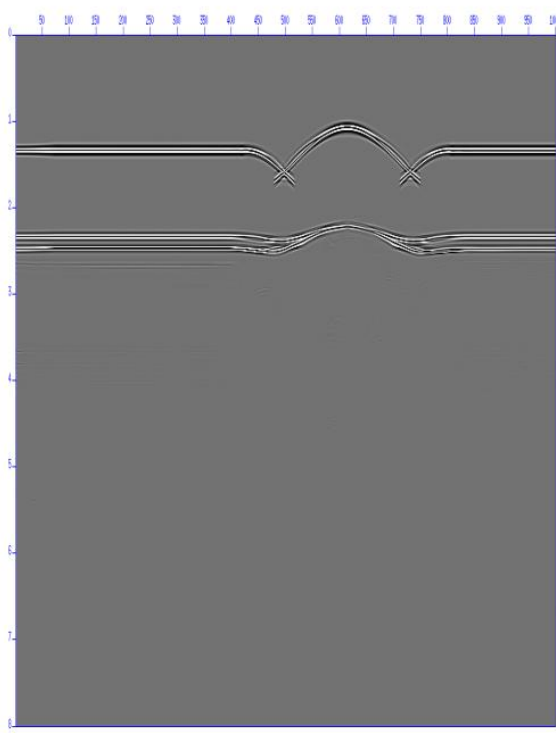

b

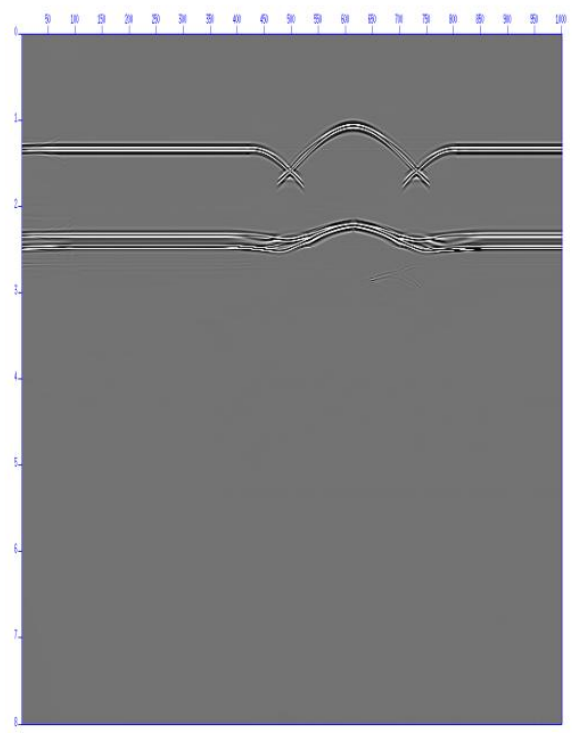

C

Fig. 6: Seção empilhada MODELO CC, a - original; b - após a aplicação da DPM; e c - após a filtragem f-k. 